\title{
Mixed Portmanteau Test for Diagnostic Checking of Time Series Models
}

\author{
Sohail Chand and Shahid Kamal \\ College of Statistical and Actuarial Sciences, University of the Punjab, Lahore, Pakistan \\ Correspondence should be addressed to Sohail Chand; sohail.stat@gmail.com
}

Received 1 April 2014; Revised 26 May 2014; Accepted 27 May 2014; Published 15 June 2014

Academic Editor: Li Weili

Copyright ( 2014 S. Chand and S. Kamal. This is an open access article distributed under the Creative Commons Attribution License, which permits unrestricted use, distribution, and reproduction in any medium, provided the original work is properly cited.

Model criticism is an important stage of model building and thus goodness of fit tests provides a set of tools for diagnostic checking of the fitted model. Several tests are suggested in literature for diagnostic checking. These tests use autocorrelation or partial autocorrelation in the residuals to criticize the adequacy of fitted model. The main idea underlying these portmanteau tests is to identify if there is any dependence structure which is yet unexplained by the fitted model. In this paper, we suggest mixed portmanteau tests based on autocorrelation and partial autocorrelation functions of the residuals. We derived the asymptotic distribution of the mixture test and studied its size and power using Monte Carlo simulations.

\section{Introduction}

In practice, there are many possible linear and nonlinear models for a problem under study, for example, autoregressive, moving average, mixed ARMA models, threshold autoregressive. Box and Jenkins [1] have described time series model building as a three-stage iterative procedure that consists of identification, estimation, and validation.

Time series models should be able to describe the dependence among the observations; see, for example, Li [2]. It is a well-discussed issue that in time series model criticism the residuals obtained from fitting a potential model to the observed time series play a vital role and can be used to detect departures from the underlying assumptions, $[1,2]$.

In particular, if the model is a good fit to the observed series then the residuals should behave somewhat like a white noise process. So, taking into account of the effect of estimation, the residuals obtained from a good fit should be approximately uncorrelated. While looking at the significance of residual autocorrelations, one approach is to test the significance of each individual residual autocorrelation which seems to be quite cumbersome. Another approach is to have some portmanteau test capable of testing the significance of the first, say, $m$, residual autocorrelations $[1,2]$, an approach we now describe.
Since Box and Pierce [3] paper, the portmanteau test has become the vital part of time series diagnostic checking. Several modifications and versions of Box and Pierce [3] have been suggested in the literature; see, for example, Ljung and Box [4], McLeod and Li [5], Monte [6], Katayama [7], and Katayama [8]. These tests are capable of testing the significance of the autocorrelations (partial autocorrelations) up to a finite number of lags.

The residuals are very commonly used as a diagnostic tool to test the goodness of fit of models. In a time series context, if the fitted model is good then it should be able to explain the dependence pattern among successive observations. In other words, all the dependence in terms of autocorrelations and partial autocorrelations of the data generating process (DGP) should be explained by the fitted model so there should be no significant autocorrelation and partial autocorrelation in successive terms of the residuals.

In practice, the most popular way for diagnostic checking of a time series model is the portmanteau test, which tests whether any of the first $m$ autocorrelations $\left(\widehat{r}_{1}, \ldots, \widehat{r}_{m}\right)$ of a time series are significantly different from zero. This type of test was first suggested by Box and Pierce [3], in which they studied the distribution of residual autocorrelations in ARIMA processes. 
Based on the autocorrelations of the residuals obtained by fitting an $\operatorname{ARMA}(p, q)$ model, Box and Pierce [3] suggested the following portmanteau test:

$$
Q_{m}=n \sum_{k=1}^{m} \widehat{r}_{k}^{2}
$$

where $\hat{r}_{k}$ is the residual autocorrelation at lag $k$. They suggested that $Q_{m} \sim \chi_{m-p-q}^{2}$, for moderate values of $m$, and the fitted model is adequate, under the following conditions:

(1) $\psi_{j} \leq O\left(n^{-1 / 2}\right)$ for $j \geq m-p$,

(2) $m / n=O\left(n^{-1 / 2}\right)$,

where $\psi_{j}$ are the weights in the $\operatorname{MA}(\infty)$ representation. This approximation requires substitution of residuals, $\widehat{\varepsilon}_{t}$, for the error term, $\varepsilon_{t}$, in the model but this sort of substitution can be a serious underestimation of significance level in diagnostic checking [9]. Many other researchers have also questioned the distribution of $Q_{m}$ (see, e.g., [10] and references therein). The choice of $m$ is an important issue.

Ljung and Box [4] suggested the use of the modified statistic

$$
Q_{m}^{*}=n(n+2) \sum_{k=1}^{m} \frac{\widehat{r}_{k}^{2}}{n-k} .
$$

They have shown that the modified portmanteau statistic $Q_{m}^{*}$ has a finite sample distribution which is much closer to $\chi_{m-p-q}^{2}$. Their results also show that $Q_{m}^{*}$ is insensitive to the normality assumption of $\varepsilon_{t}$. As pointed out by many researchers, for example, Davies et al. [11], Ansley and Newbold [12], the true significance levels of $Q_{m}$ tend to be much lower than predicted by the asymptotic theory and though the mean of $Q_{m}^{*}$ is much closer to the asymptotic distribution, this corrected version of the portmanteau test has an inflated variance. But Ljung and Box [4] pointed out that approximate expression of variance given by Davies et al. [11] overestimates the variance of $Q_{m}^{*}$.

McLeod [10, Theorem 1] has shown that $\widehat{\mathbf{r}}=\left(\widehat{r}_{1}, \ldots, \widehat{r}_{m}\right)$ is approximately normal with mean $\mathbf{0}$ and $\operatorname{Var}(\widehat{\mathbf{r}})=(\mathbf{I}-$ $\mathbf{C )} / n$, where $\mathbf{C}=\mathbf{X} \rrbracket^{-1} \mathbf{X}^{T}$, $\mathbf{I}$ is the identity matrix, and $\square$ is the Fisher information matrix. The superscript $T$ stands for transposition of matrix. We noticed that approximation of $\mathbf{C}$ by $\mathbf{D}=\mathbf{X}\left(\mathbf{X}^{T} \mathbf{X}\right)^{-1} \mathbf{X}^{T}$, especially when $m$ is small, is a source of bias in approximating the asymptotic distribution of portmanteau tests.

In practice, the optimal choice of $m$ is difficult as the use of the $\chi_{m-p-q}^{2}$ approximation and diagnostic checking require large values of $m$ which results in less power and unstable size of test, as noticed by Ljung [13] and Katayama [7]. Katayama [8] suggested a multiple portmanteau test to overcome this problem. His suggested test is based on several portmanteau tests for a range of small to medium values of $m$. He showed using some numerical examples that his suggestion leads to a superior test. He also discussed the linkage between his suggested multiple test and the test due to Peña and Rodríguez [14]. He suggested a method based on some iterative procedure to approximate joint distribution of the multiple test as the computation of the distribution is very hard due to correlated elements.

The portmanteau tests $Q_{m}$ and $Q_{m}^{*}$ are based on the autocorrelations. Monti [6] suggested a portmanteau test

$$
Q_{m}^{*}(\widehat{\omega})=n(n+2) \sum_{k=1}^{m} \frac{\widehat{\omega}_{k}^{2}}{n-k}
$$

where $\widehat{\omega}_{k}$ is the residual partial autocorrelation at lag $k$. She showed that $Q_{m}^{*}(\widehat{\omega})$, analogously to $Q_{m}^{*}$, has an asymptotic null distribution $\chi_{m-p-q}^{2}$ and that $Q_{m}^{*}(\widehat{\omega})$ is more powerful than $Q_{m}^{*}$ especially when the order of the moving average component is understated. Chand and Kamal [15] had studied the size of Monti's test.

Portmanteau tests, for example, Box-Pierce test, which are based on residuals autocorrelations, have good power against the understatement of autoregressive component while the tests using residuals partial autocorrelations have shown good power against the understatement of moving average term. A new test using both autocorrelation and partial autocorrelations is expected to perform better in both of these two scenarios and will be capable of picking up understatement in moving average and autoregressive components.

\section{Materials and Methods}

In this section, we give the algorithms for the Monte Carlo method used to compute the empirical size and power of the diagnostic tests defined in Section 2. For each Monte Carlo run, a sample time series $\left\{y_{t}\right\}_{t=1}^{n}$ is simulated under the model $\mathscr{M}$. For empirical size, $\mathscr{M}$ is the null model while for the computation of power it is the alternative model. In both of the situations, we estimate the null model for the simulated sample time series and $T$ is calculated from the residuals, $\widehat{\varepsilon}_{t}$.

Algorithm 1 gives the bootstrap procedure used in our numerical study. From this algorithm, we obtain the bootstrap approximation of the distribution of the test. We will use this algorithm to compute empirical size in the following algorithm of our simulation study consisting of $N$ Monte Carlo runs.

\section{Results}

Suppose we have an observed time series $\left\{y_{t}: t=1,2, \ldots, n\right\}$ generated by an $\operatorname{ARMA}(p, q)$ model given by

$$
\phi(B) y_{t}=\theta(B) \varepsilon_{t}
$$

where $B$ is the backward shift operator, $\phi(B)=1-\phi_{1} B-$ $\phi_{2} B^{2}-\cdots-\phi_{p} B^{p}$ is the autoregressive polynomial of order $p$, and $\theta(B)=1+\theta_{1} B+\theta_{2} B^{2}+\cdots+\theta_{q} B^{q}$ is the moving average polynomial of order $q$. Let an appropriately identified model be fitted to the observed time series and let residuals $\widehat{\varepsilon}_{1}, \widehat{\varepsilon}_{2}, \ldots, \widehat{\varepsilon}_{n}$ be obtained. These residuals play an important 
Step 1. Generate bootstrap sample $y_{t}^{*}$ using resamples of residuals, $\widehat{\varepsilon}_{t}$, obtained by fitting the model under null hypothesis to the simulated time series. We denoted these resampled residuals as $\varepsilon_{t}^{*}$.

Step 2. Fit the null model to the bootstrap sample $y_{t}^{*}$ and obtain residuals as $\widehat{\varepsilon}_{t}^{*}=y_{t}^{*}-\widehat{y}_{t}^{*}$, where $\widehat{y}_{t}^{*}$ is the fitted series.

Step 3. Using the residuals, $\widehat{\varepsilon}_{t}^{*}$, obtained in Step 2 above, calculate test-statistic $T$. We denote it as $T^{*}$.

Step 4. Repeat Step 1-3 for each of the $B$ bootstrap samples.

Algorithm 1: Bootstrap sampling procedure.

role in the diagnostic checking of the fitted model. The residuals autocorrelation at lag $k$ can be defined as

$$
\widehat{r}_{k}=\frac{\sum_{t=k+1}^{n} \widehat{\varepsilon}_{t} \widehat{\varepsilon}_{t-k}}{\sum_{t=1}^{n} \widehat{\varepsilon}_{t}^{2}} \quad k=1,2, \ldots,
$$

where $\left\{\widehat{\varepsilon}_{t-k}\right\}$ is the series at lag $k$ of $\left\{\widehat{\varepsilon}_{t}\right\}$. Let $\boldsymbol{\beta}=$ $\left(\phi_{1}, \ldots, \phi_{p}, \theta_{1}, \ldots, \theta_{q}\right)$ be the vector of model parameters and let $\widehat{\boldsymbol{\beta}}=\left(\widehat{\phi}_{1}, \ldots, \widehat{\phi}_{p}, \widehat{\theta}_{1}, \ldots, \widehat{\theta}_{q}\right)$ be the corresponding estimator of $\beta$.

Let us define autocorrelation of error term, $\varepsilon_{t}$, that is, white noise at lag $k$, as

$$
r_{k}=\frac{\sum_{t=k+1}^{n} \varepsilon_{t} \varepsilon_{t-k}}{\sum_{t=1}^{n} \varepsilon_{t}^{2}} \quad k=1,2, \ldots
$$

The root mean square error of $\widehat{\boldsymbol{\beta}}$ can be defined as

$$
\sqrt{E(\boldsymbol{\beta}-\widehat{\boldsymbol{\beta}})^{2}} .
$$

As the above term is of order $1 / \sqrt{n}$, thus we can use Taylor series of first order to approximate $\widehat{r}_{k}$ around $r_{k}$. We can write

$$
\widehat{r}_{k}=r_{k}+\sum_{j=1}^{p+q}\left(\beta_{j}-\widehat{\beta}_{j}\right)^{2} \lambda_{j k}+O_{p}\left(\frac{1}{n}\right),
$$

where

$$
\begin{aligned}
& \lambda_{j k}=-\left.\frac{\partial r_{k}}{\partial \dot{\beta}_{j}}\right|_{\dot{\beta}=\widehat{\beta}}, \\
& \hat{\lambda}_{j k}=-\left.\frac{\partial \widehat{r}_{k}}{\partial \dot{\beta}_{j}}\right|_{\dot{\beta}=\hat{\beta}} .
\end{aligned}
$$

Take

$$
\varepsilon_{t}=y_{t}-\phi_{1} y_{t-1}-\cdots-\phi_{p} y_{t-p}-\theta_{1} \varepsilon_{t-1}-\cdots-\theta_{q} \varepsilon_{t-q} .
$$

For an $\operatorname{AR}(p)$ process

$$
\varepsilon_{t}=y_{t}-\phi_{1} y_{t-1}-\cdots-\phi_{p} y_{t-p},
$$

at $\boldsymbol{\beta}=\dot{\boldsymbol{\beta}}$, that is, for an $\operatorname{AR}(p), \boldsymbol{\phi}=\dot{\boldsymbol{\phi}}$, we can write

$$
\begin{gathered}
\dot{\varepsilon}_{t}=y_{t}-\dot{\phi}_{1} y_{t-1}-\cdots-\dot{\phi}_{p} y_{t-p}, \\
\frac{\partial \dot{\varepsilon}_{t}}{\partial \dot{\phi}_{j}}=-y_{t-j} .
\end{gathered}
$$

From (10) and summing over observed sample, we get

$$
\sum \varepsilon_{t}^{2}=\sum\left(y_{t}-\phi_{1} y_{t-1} \cdots-\phi_{p} y_{t-p}\right)^{2} .
$$

Partially differentiating with respect to $\phi_{j}$

$$
\frac{\partial \sum \varepsilon_{t}^{2}}{\partial \phi_{j}}=2 \sum\left(y_{t}-\phi_{1} y_{t-1} \cdots-\phi_{p} y_{t-p}\right)\left(-y_{t-j}\right) .
$$

At $\phi=\dot{\phi}$

$$
\frac{\partial \sum \varepsilon_{t}^{2}}{\partial \phi_{j}}=0
$$

Now

$$
\begin{aligned}
\widehat{\lambda}_{j k} & =-\frac{\partial \widehat{r}_{k}}{\partial \dot{\phi}_{j}}=-\frac{\partial\left(c_{k} / c_{0}\right)}{\partial \phi_{j}} \\
& =-\frac{\partial}{\partial \phi_{j}}\left(\frac{\sum \varepsilon_{t} \varepsilon_{t-k}}{\sum \varepsilon_{t}^{2}}\right)=-\frac{1}{\sum \varepsilon_{t}^{2}} \frac{\partial\left(\sum \varepsilon_{t} \varepsilon_{t-k}\right)}{\partial \phi_{j}} .
\end{aligned}
$$

As

$$
\sum \varepsilon_{t} \varepsilon_{t-k}=\sum\left(\phi(B) y_{t}\right)\left(\phi(B) y_{t-k}\right)
$$

$$
\begin{gathered}
=\sum_{t}\left(\sum_{\substack{i=j \\
i=k+j}} \phi_{i} \phi_{j} y_{t-i} y_{t-k-j}\right. \\
\left.+\sum_{\substack{i \\
i \neq k+j}} \phi_{i} \phi_{j} y_{t-i} y_{t-k-j}\right),
\end{gathered}
$$

thus

$$
\begin{aligned}
\widehat{\lambda}_{j k} & =-\frac{\sum_{t} y_{t}^{2}}{\sum_{t} \widehat{\varepsilon}_{t}^{2}} \sum_{i}\left(r_{k-i+j}(y)+r_{k+i-j}(y)\right) \\
& =-\frac{\sum_{i} p \widehat{h} i_{i}\left(r_{k-i+j}(y)+r_{k+i-j}(y)\right)}{\sum_{i} \sum_{j} \widehat{\phi}_{i} \widehat{\phi}_{j} r_{i-j}(y)},
\end{aligned}
$$

where

$$
r_{k}(y)=\frac{\sum y_{t} y_{t-k}}{\sum y_{t}^{2}}
$$


Using Bartlett's formula, we can write

$$
r_{k}(y)=\rho_{k}+O_{p}\left(n^{-1 / 2}\right) .
$$

Using $\widehat{\phi}_{j}=\phi_{j}+O_{p}\left(n^{-1 / 2}\right)$ and the above results

$$
\hat{\lambda}_{j k}=\lambda_{j k}+O_{p}\left(n^{-1 / 2}\right) .
$$

Consider the $\operatorname{AR}(p)$ process

$$
\begin{gathered}
\phi(B) y_{t}=\varepsilon_{t}, \\
\left(1-\phi_{1} B-\phi_{2} B^{2}-\cdots-\phi_{p} B^{p}\right) y_{t}=\varepsilon_{t}, \\
y_{t}-\phi_{1} y_{t-1}-\phi_{2} y_{t-2}-\cdots-\phi_{p} y_{t-p}=\varepsilon_{t} .
\end{gathered}
$$

Multiplying both sides by $y_{t-k}$ and on taking expectation, we get $E\left(y_{t} y_{t-k}\right)-\phi_{1} E\left(y_{t-1} y_{t-k}\right)-\phi_{2} E\left(y_{t-2} y_{t-k}\right)-\cdots-$ $\phi_{p} E\left(y_{t-p} y_{t-k}\right)=E\left(\varepsilon_{t} y_{t-k}\right)$. Without loss of generality, we can assume $E\left(y_{t}\right)=0$; moreover it is considered a stationary process; therefore $E\left(y_{t}^{2}\right)=\operatorname{var}\left(y_{t}\right)=c_{0}$ and $E\left(y_{t} y_{t-k}\right)=$ $\operatorname{cov}\left(y_{t}, y t-k\right)=c_{k}$. Moreover, $E\left(\varepsilon_{t} y_{t-k}\right)=0$ for $k \geq 1$. Thus

$$
c_{k}-\phi_{1} c_{k-1}-\phi_{2} c_{k-2}-\cdots-\phi_{p} c_{k-p}=0
$$

Divide both sides by $c_{0}$ and using $r_{k}=c_{k} / c_{0}$, we get

$$
\begin{gathered}
r_{k}-\phi_{1} r_{k-1}-\phi_{2} r_{k-2}-\cdots-\phi_{p} r_{k-p}=0, \\
\phi(B) r_{k}=0 .
\end{gathered}
$$

Using this result and the structure of $\operatorname{AR}(p)$ process, it implies

$$
\lambda_{j k}=\frac{\sum_{i} \phi_{i} \phi_{k-j+i}}{\sum_{i} \phi_{i} \rho_{i}} .
$$

The $\operatorname{AR}(p)$ process can be written in an equivalent moving average representation, that is, $\mathrm{MA}(\infty)$, given as

$$
\begin{aligned}
& \phi(B) y_{t}=\varepsilon_{t}, \\
& y_{t}=\psi(B) \varepsilon_{t},
\end{aligned}
$$

where

$$
\psi(B)=\frac{1}{\phi(B)}=\sum_{i=0}^{\infty} \psi_{i} B^{i} .
$$

Using Box and Pierce [3, page. 1514] and the above results, we can write

$$
\lambda_{j k}=\psi_{k-j},
$$

where $k=1,2, \ldots, m$, and thus using (8), we get

$$
\widehat{r}_{k}=r_{k}+\sum_{i}\left(\phi_{i}-\widehat{\phi}_{i}\right) \psi_{k-i}+O_{p}\left(\frac{1}{n}\right) \text {. }
$$

Using McLeod [10, Theorem 1], the asymptotic distribution of $\widehat{\mathbf{r}}=\left(\widehat{r}_{1}, \ldots, \widehat{r}_{m}\right)$, where $m \geq p+q$ is normal with mean $\mathbf{0}$ and covariance matrix $\left(\mathbf{1}-\mathbf{X} \rrbracket^{-1} \mathbf{X}^{T}\right) / n$; that is,

$$
\widehat{\mathbf{r}} \sim N\left(\mathbf{0}, \frac{\left(\mathbf{I}-\mathbf{X} \rrbracket^{-1} \mathbf{X}^{T}\right)}{n}\right),
$$

where for an $\operatorname{ARMA}(p, q)$ process

$$
\mathbf{X}=\left[\begin{array}{cccc|cccc}
1 & 0 & \ldots & 0 & 1 & 0 & \ldots & 0 \\
\psi_{1} & 1 & \ldots & 0 & \pi_{1} & 1 & \ldots & 0 \\
\psi_{2} & \psi_{1} & \ldots & 0 & \pi_{2} & \pi_{1} & \ldots & 0 \\
\vdots & \vdots & & \vdots & \vdots & \vdots & & \vdots \\
\psi_{m-1} & \psi_{m-2} & \ldots & \psi_{m-p} & \pi_{m-1} & \pi_{m-2} & \ldots & \pi_{m-p}
\end{array}\right]
$$

The elements $\psi_{j}$ and $\pi_{j}$ are the coefficients of $B^{j}$ in the infinite expansion of inverse of AR and MA polynomials. For an $\operatorname{AR}(p)$ process, the above matrix reduces to

$$
\mathbf{X}=\left[\begin{array}{cccc}
1 & 0 & \cdots & 0 \\
\psi_{1} & 1 & \cdots & 0 \\
\psi_{2} & \psi_{1} & \cdots & 0 \\
\vdots & \vdots & & \vdots \\
\psi_{m-1} & \psi_{m-2} & \cdots & \psi_{m-p}
\end{array}\right]
$$

Let $\omega_{k}$ be the partial autocorrelation at lag $k$; then using Monti [6, page 777 , equation (4)], we have

$$
\widehat{\boldsymbol{\omega}}=\widehat{\mathbf{r}}+O_{p}\left(\frac{1}{n}\right) .
$$

According to Monti [6], the $\widehat{\mathbf{r}}$ can be obtained using a singular linear transformation of the noise autocorrelations, that is, $\mathbf{r}$, given as

$$
\widehat{\mathbf{r}}=\left(\mathbf{I}-\mathbf{X}\left(\mathbf{X}^{T} \mathbf{X}\right)^{-1} \mathbf{X}\right) \mathbf{r}+O_{p}\left(\frac{1}{n}\right) .
$$

Using (30), we can write

$$
\widehat{\boldsymbol{\omega}} \sim N\left(\mathbf{0}, \frac{\left(\mathbf{I}-\mathbf{X} \rrbracket^{-1} \mathbf{X}^{T}\right)}{n}\right) .
$$

Ljung and Box [4], using (30), suggested a portmanteau test for diagnostic testing of the fitted $\operatorname{ARMA}(p, q)$ model given as

$$
Q_{m}^{*}(\widehat{r})=n(n+2) \sum_{k=1}^{m} \frac{\widehat{r}_{k}^{2}}{n-k} \sim \chi_{m-p-q}^{2} .
$$

Following the same lines and using the result in (35), Monti [6] suggested the following portmanteau test:

$$
Q_{m}^{*}(\widehat{\omega})=n(n+2) \sum_{k=1}^{m} \frac{\widehat{\omega}_{k}^{2}}{n-k} \sim \chi_{m-p-q}^{2} .
$$

\section{New Test}

We suggest a new test $Q_{m}^{\dagger}(\widehat{r}, \widehat{\omega})=Q_{m}^{*}(\widehat{r})+Q_{m}^{*}(\widehat{\omega})$, that is, the sum of Ljung and Box [4] and Monti [6], given as

$$
Q_{m}^{*}(\widehat{r}, \widehat{\omega})=n(n+2) \sum_{k=1}^{m} \frac{\left(\widehat{r}_{k}^{2}+\widehat{\omega}_{k}^{2}\right)}{n-k} .
$$


TABle 1: Monte Carlo estimates of $\rho_{m}$.

\begin{tabular}{lcccccccc}
\hline$n$ & & & & $m$ & & \\
& 2 & 3 & 5 & 10 & 15 & 20 & -25 \\
\hline 8 & 0.799 & - & - & - & - & - & - & - \\
12 & 0.882 & 0.872 & - & - & - & - & - \\
20 & 0.932 & 0.909 & 0.851 & - & - & - & - \\
40 & 0.962 & 0.947 & 0.910 & 0.829 & - & - & - \\
60 & 0.974 & 0.968 & 0.934 & 0.876 & 0.828 & - & - \\
80 & 0.982 & 0.974 & 0.951 & 0.898 & 0.859 & 0.825 & 0.851 & - \\
100 & 0.986 & 0.979 & 0.958 & 0.923 & 0.881 & 0.873 & 0.851 & 0.829 \\
125 & 0.989 & 0.983 & 0.968 & 0.936 & 0.902 & 0.873 & 0.854 \\
150 & 0.990 & 0.983 & 0.972 & 0.941 & 0.920 & 0.887 & 0.875 \\
200 & 0.993 & 0.988 & 0.979 & 0.956 & 0.934 & 0.915 & 0.900 & 0.874 \\
\hline
\end{tabular}

TABLE 2: Empirical size at 1\% nominal size for an AR(1) process: $y_{t}=\phi y_{t-1}+\varepsilon_{t}$.

\begin{tabular}{|c|c|c|c|c|c|c|}
\hline \multirow{2}{*}{$m$} & \multirow{2}{*}{$T$} & \multicolumn{5}{|c|}{$\phi$} \\
\hline & & 0.1 & 0.4 & 0.7 & 0.8 & 0.9 \\
\hline \multirow{3}{*}{5} & $Q_{m}^{*}(\widehat{r})$ & 0.021 & 0.020 & 0.027 & 0.024 & 0.027 \\
\hline & $Q_{m}^{*}(\widehat{\omega})$ & 0.012 & 0.011 & 0.010 & 0.005 & 0.015 \\
\hline & $Q_{m}^{*}(\widehat{r}, \widehat{\omega})$ & 0.014 & 0.015 & 0.016 & 0.007 & 0.020 \\
\hline \multirow{3}{*}{10} & $Q_{m}^{*}(\widehat{r})$ & 0.008 & 0.015 & 0.016 & 0.017 & 0.022 \\
\hline & $Q_{m}^{*}(\widehat{\omega})$ & 0.007 & 0.012 & 0.006 & 0.009 & 0.018 \\
\hline & $Q_{m}^{*}(\widehat{r}, \widehat{\omega})$ & 0.006 & 0.011 & 0.008 & 0.010 & 0.017 \\
\hline \multirow{3}{*}{15} & $Q_{m}^{*}(\widehat{r})$ & 0.013 & 0.025 & 0.020 & 0.018 & 0.025 \\
\hline & $Q_{m}^{*}(\widehat{\omega})$ & 0.006 & 0.010 & 0.007 & 0.010 & 0.015 \\
\hline & $Q_{m}^{*}(\widehat{r}, \widehat{\omega})$ & 0.007 & 0.013 & 0.010 & 0.010 & 0.015 \\
\hline \multirow{3}{*}{20} & $Q_{m}^{*}(\widehat{r})$ & 0.024 & 0.022 & 0.022 & 0.022 & 0.034 \\
\hline & $Q_{m}^{*}(\widehat{\omega})$ & 0.011 & 0.011 & 0.011 & 0.012 & 0.009 \\
\hline & $Q_{m}^{*}(\widehat{r}, \widehat{\omega})$ & 0.011 & 0.015 & 0.018 & 0.014 & 0.019 \\
\hline \multirow{3}{*}{25} & $Q_{m}^{*}(\widehat{r})$ & 0.021 & 0.020 & 0.027 & 0.024 & 0.027 \\
\hline & $Q_{m}^{*}(\widehat{\omega})$ & 0.012 & 0.011 & 0.010 & 0.005 & 0.015 \\
\hline & $Q_{m}^{*}(\widehat{r}, \widehat{\omega})$ & 0.014 & 0.015 & 0.016 & 0.007 & 0.018 \\
\hline
\end{tabular}

TABLE 3: Empirical size at 5\% nominal size for an AR(1) process: $y_{t}=\phi y_{t-1}+\varepsilon_{t}$.

\begin{tabular}{|c|c|c|c|c|c|c|}
\hline \multirow{2}{*}{$m$} & \multirow{2}{*}{$T$} & \multicolumn{5}{|c|}{$\phi$} \\
\hline & & 0.1 & 0.4 & 0.7 & 0.8 & 0.9 \\
\hline \multirow{3}{*}{5} & $Q_{m}^{*}(\widehat{r})$ & 0.077 & 0.063 & 0.078 & 0.072 & 0.085 \\
\hline & $Q_{m}^{*}(\widehat{\omega})$ & 0.050 & 0.051 & 0.053 & 0.047 & 0.055 \\
\hline & $Q_{m}^{*}(\widehat{r}, \widehat{\omega})$ & 0.056 & 0.049 & 0.055 & 0.049 & 0.052 \\
\hline \multirow{3}{*}{10} & $Q_{m}^{*}(\widehat{r})$ & 0.041 & 0.053 & 0.057 & 0.059 & 0.077 \\
\hline & $Q_{m}^{*}(\widehat{\omega})$ & 0.044 & 0.053 & 0.059 & 0.056 & 0.07 \\
\hline & $Q_{m}^{*}(\widehat{r}, \widehat{\omega})$ & 0.036 & 0.046 & 0.045 & 0.049 & 0.061 \\
\hline \multirow{3}{*}{15} & $Q_{m}^{*}(\widehat{r})$ & 0.06 & 0.069 & 0.072 & 0.074 & 0.089 \\
\hline & $Q_{m}^{*}(\widehat{\omega})$ & 0.052 & 0.065 & 0.055 & 0.057 & 0.068 \\
\hline & $Q_{m}^{*}(\widehat{r}, \widehat{\omega})$ & 0.053 & 0.061 & 0.051 & 0.049 & 0.055 \\
\hline \multirow{3}{*}{20} & $Q_{m}^{*}(\widehat{r})$ & 0.065 & 0.07 & 0.059 & 0.087 & 0.085 \\
\hline & $Q_{m}^{*}(\widehat{\omega})$ & 0.051 & 0.054 & 0.056 & 0.064 & 0.061 \\
\hline & $Q_{m}^{*}(\widehat{r}, \widehat{\omega})$ & 0.053 & 0.051 & 0.049 & 0.065 & 0.072 \\
\hline \multirow{3}{*}{25} & $Q_{m}^{*}(\widehat{r})$ & 0.077 & 0.063 & 0.078 & 0.072 & 0.085 \\
\hline & $Q_{m}^{*}(\widehat{\omega})$ & 0.050 & 0.051 & 0.053 & 0.047 & 0.055 \\
\hline & $Q_{m}^{*}(\widehat{r}, \widehat{\omega})$ & 0.056 & 0.049 & 0.055 & 0.049 & 0.062 \\
\hline
\end{tabular}


Step 1. Obtain $T^{*}$ using Algorithm 1, for each of the $B$ bootstrap samples, reject null model if $T^{*} \geq T$, otherwise accept it.

Step 2. Determine the proportion of $B$ bootstrap samples, say $\widehat{p}_{c}$, for which the null hypothesis is rejected.

Step 3. Repeat Step 1-2 for each of the N Monte Carlo runs.

Step 4. Empirical size, $\widehat{\alpha}$, is determined as the proportion of Monte Carlo runs for which the $\widehat{p}_{c} \leq \alpha$, where $\alpha$ is the level of significance,

$$
\widehat{\alpha}=\frac{\#\left(\hat{p}_{c} \leq \alpha\right)}{N} .
$$

Algorithm 2: Computation of empirical size.

Step 1. Calculate $100(1-\alpha)$ th percentile, say $T_{1-\alpha}^{*}$, of the bootstrap distribution of $T^{*}$ obtained using Algorithm 1.

Step 2. Reject null model if $T \geq T_{1-\alpha}^{*}$ otherwise accept it.

Step 3. Repeat Step 1-2 for each of the $N$ Monte Carlo runs.

Step 4. Empirical power, $1-\widehat{\beta}$, is determined as below,

$$
1-\widehat{\beta}=\frac{\#\left(T \geq T_{1-\alpha}\right)}{N} \text {. }
$$

Algorithm 3: Computation of empirical power.

It is obvious that this new statistic $Q_{m}^{*}$ is the sum of two chi-square variables as discussed above in (36) and (37). Moreover, it can be noticed that both of these random variables will be positively correlated due to their structure of using residual autocorrelation and partial autocorrelation. We have discussed their correlational structure later in this report.

Using the result of Joarder and Omar [16, Theorem 3.1], we can write the probability density function of $Z=Q_{m}^{*}=$ $Q_{m}(\widehat{r})+Q_{m}(\widehat{\omega})$ as

$$
\begin{gathered}
f_{Z}(z)=\frac{\left(1-\rho_{m}^{2}\right)^{-m / 2}}{2^{m} \Gamma(m)} z^{m-1} \exp \left(\frac{-z}{2-2 \rho_{m}^{2}}\right) \\
\times{ }_{0} F_{1}\left(\frac{m+1}{2} ; \frac{\rho_{m}^{2} z^{2}}{\left(4-4 \rho_{m}^{2}\right)^{2}}\right) ; \\
0 \leq z<\infty,
\end{gathered}
$$

where $\rho_{m}$ is the correlation between $Q_{m}^{*}(\widehat{r})$ and $Q_{m}^{*}(\widehat{\omega})$ :

$$
\begin{gathered}
{ }_{p} F_{q}\left(a_{1}, a_{2}, \ldots, a_{p} ; b_{1}, b_{2}, \ldots, b_{q} ; z\right)=\sum_{i=0}^{\infty} \frac{\prod_{j=1}^{p}\left(a_{j}\right)_{\{i\}} z^{i}}{\prod_{j=1}^{q}\left(b_{j}\right)_{\{i\}} i !} \\
a_{\{i\}}=a(a+1) \cdots(a+i-1) .
\end{gathered}
$$

Now the important issue is to estimate $\rho_{m}$ which is discussed in Section 4.1.

4.1. Estimation of $\rho_{m}$. We have used Monte Carlo simulation to estimate $\rho_{m}$. We have considered various choices of sample size and $m$ and obtained the following results. Empirical results suggest that, for any $n$, we have

$$
0.8 \leq \rho_{m} \leq 1,
$$

where $m \leq n / 4$. Results are given in Table 1 .

4.2. Empirical Size. The size of a test is helpful in assessing how reasonable is our assumption of the null distribution. We can compute the size when the sample is simulated under the null model. This is the probability of rejecting the null model when the null model is true model. The model under null hypothesis is considered an $\mathrm{AR}(1)$ process $y_{t}=\phi y_{t-1}+\varepsilon_{t}$. To see the effect of stationarity and near to unit-root problem, we have considered various choices of $\phi$. The value of $\phi$ near to 1 , for example, $\phi=0.9$, is an example of near nonstationary process.

It can be seen from the results shown in Tables 2 and 3 that our new suggested mixture test, that is, $Q_{m}^{*}(\widehat{r}, \widehat{\omega})$, have close approximation of corresponding nominal sizes. These results show the good approximation of asymptotic distribution of our new suggested test.

4.3. Empirical Power. The power of a test is the probability of rejecting a false null hypothesis. For empirical power, as mentioned earlier, the sample is generated under the alternative model. Algorithms 2 and 3 state the Monte Carlo procedure we use to determine the empirical size and power of test.

For power computation, we have simulated under an $\operatorname{ARMA}(1,1)$ process $y_{t}=\phi y_{t-1}+\varepsilon_{t}+0.8 \varepsilon_{t-1}$ and $y_{t}=$ $0.8 y_{t-1}+\varepsilon_{t}+\theta \varepsilon_{t-1}$ as alternative models, while $\mathrm{AR}(1)$ process $y_{t}=\phi y_{t-1}+\varepsilon_{t}$ is the model specified in null hypothesis. Results are provided in Tables 4 and 5. These results show that our suggested mixed portmanteau tests have empirical power 
TABLE 4: Empirical power by fitting an $\operatorname{AR}(1)$ process against an $\operatorname{ARMA}(1,1)$ process: $y_{t}=\phi y_{t-1}+\varepsilon_{t}+0.8 \varepsilon_{t-1}$ using 1000 Monte Carlo runs.

\begin{tabular}{|c|c|c|c|c|c|c|}
\hline \multirow{2}{*}{$m$} & \multirow{2}{*}{$T$} & \multicolumn{5}{|c|}{$\phi$} \\
\hline & & 0.1 & 0.4 & 0.7 & 0.8 & 0.9 \\
\hline \multirow{3}{*}{5} & $Q_{m}^{*}(\widehat{r})$ & 0.955 & 0.991 & 1 & 1 & 1 \\
\hline & $Q_{m}^{*}(\widehat{\omega})$ & 0.992 & 0.997 & 1 & 1 & 1 \\
\hline & $Q_{m}^{*}(\widehat{r}, \widehat{\omega})$ & 0.997 & 0.999 & 1 & 1 & 1 \\
\hline \multirow{3}{*}{10} & $Q_{m}^{*}(\widehat{r})$ & 0.819 & 0.941 & 0.983 & 0.986 & 0.995 \\
\hline & $Q_{m}^{*}(\widehat{\omega})$ & 0.980 & 1 & 1 & 1 & 1 \\
\hline & $Q_{m}^{*}(\widehat{r}, \widehat{\omega})$ & 0.985 & 1 & 1 & 1 & 1 \\
\hline \multirow{3}{*}{15} & $Q_{m}^{*}(\widehat{r})$ & 0.748 & 0.871 & 0.940 & 0.957 & 0.976 \\
\hline & $Q_{m}^{*}(\widehat{\omega})$ & 0.958 & 0.990 & 1 & 1 & 1 \\
\hline & $Q_{m}^{*}(\widehat{r}, \widehat{\omega})$ & 0.953 & 0.970 & 0.995 & 0.997 & 1 \\
\hline \multirow{3}{*}{20} & $Q_{m}^{*}(\widehat{r})$ & 0.680 & 0.828 & 0.900 & 0.940 & 0.948 \\
\hline & $Q_{m}^{*}(\widehat{\omega})$ & 0.926 & 0.989 & 0.995 & 0.999 & 1 \\
\hline & $Q_{m}^{*}(\widehat{r}, \widehat{\omega})$ & 0.943 & 0.957 & 0.987 & 0.998 & 0.999 \\
\hline \multirow{3}{*}{25} & $Q_{m}^{*}(\widehat{r})$ & 0.614 & 0.810 & 0.872 & 0.910 & 0.934 \\
\hline & $Q_{m}^{*}(\widehat{\omega})$ & 0.864 & 0.974 & 0.990 & 0.999 & 1 \\
\hline & $Q_{m}^{*}(\widehat{r}, \widehat{\omega})$ & 0.927 & 0.940 & 0.975 & 0.995 & 0.998 \\
\hline
\end{tabular}

TABLE 5: Empirical power by fitting an $\operatorname{AR}(1)$ process against an $\operatorname{ARMA}(1,1)$ process: $y_{t}=0.8 y_{t-1}+\varepsilon_{t}+\theta \varepsilon_{t-1}$ using 1000 Monte Carlo runs.

\begin{tabular}{|c|c|c|c|c|c|c|}
\hline \multirow{2}{*}{$m$} & \multirow{2}{*}{$T$} & \multicolumn{5}{|c|}{$\theta$} \\
\hline & & 0.1 & 0.4 & 0.7 & 0.8 & 0.9 \\
\hline \multirow{3}{*}{5} & $Q_{m}^{*}(\widehat{r})$ & 0.103 & 0.703 & 1 & 1 & 1 \\
\hline & $Q_{m}^{*}(\widehat{\omega})$ & 0.081 & 0.771 & 1 & 1 & 1 \\
\hline & $Q_{m}^{*}(\widehat{r}, \widehat{\omega})$ & 0.208 & 0.846 & 1 & 1 & 1 \\
\hline \multirow{3}{*}{10} & $Q_{m}^{*}(\widehat{r})$ & 0.092 & 0.520 & 0.917 & 1 & 0.978 \\
\hline & $Q_{m}^{*}(\widehat{\omega})$ & 0.115 & 0.601 & 1 & 1 & 1 \\
\hline & $Q_{m}^{*}(\widehat{r}, \widehat{\omega})$ & 0.228 & 0.784 & 1 & 1 & 1 \\
\hline \multirow{3}{*}{15} & $Q_{m}^{*}(\widehat{r})$ & 0.063 & 0.554 & 0.941 & 0.963 & 0.955 \\
\hline & $Q_{m}^{*}(\widehat{\omega})$ & 0.051 & 0.552 & 1 & 1 & 1 \\
\hline & $Q_{m}^{*}(\widehat{r}, \widehat{\omega})$ & 0.196 & 0.747 & 1 & 1 & 1 \\
\hline \multirow{3}{*}{20} & $Q_{m}^{*}(\widehat{r})$ & 0.094 & 0.441 & 0.832 & 0.964 & 0.907 \\
\hline & $Q_{m}^{*}(\widehat{\omega})$ & 0.063 & 0.491 & 1 & 1 & 1 \\
\hline & $Q_{m}^{*}(\widehat{r}, \widehat{\omega})$ & 0.187 & 0.698 & 1 & 1 & 1 \\
\hline \multirow{3}{*}{25} & $Q_{m}^{*}(\widehat{r})$ & 0.020 & 0.475 & 0.801 & 0.896 & 0.858 \\
\hline & $Q_{m}^{*}(\widehat{\omega})$ & 0.053 & 0.571 & 0.982 & 1 & 1 \\
\hline & $Q_{m}^{*}(\widehat{r}, \widehat{\omega})$ & 0.176 & 0.669 & 0.994 & 1 & 1 \\
\hline
\end{tabular}

comparable with other tests. It can be noticed that our new test has shown power better than other considered tests when moving average part is understated and the moving average parameter is relatively small.

\section{Conclusion}

Portmanteau tests based on residuals autocorrelations and partial autocorrelations have their individual good properties. Our suggested mixture portmanteau tests have a good approximation of their asymptotic distribution. As it is important to study both the residuals autocorrelation and the partial autocorrelations for model diagnostic checking, so this new test is capable of doing it simultaneously instead of running separate diagnostic tests.

\section{Conflict of Interests}

The authors declare that there is no conflict of interests regarding the publication of this paper.

\section{Acknowledgments}

The authors thank the editor and the referees for their helpful comments and suggestions that greatly improved the previous version of this paper. The work of first author was 
supported by the Research Grant 5227-Est-I-2013 from the University of the Punjab, Lahore, Pakistan.

\section{References}

[1] G. Box and G. Jenkins, Time Series Analysis, Forecasting and Control, John Wiley \& Sons, New Jersey, NJ, USA, 1994.

[2] W. Li, Diagnostic Checks in Time Series, Chapman \& Hall/CRC, New York, NY, USA, 2004.

[3] G. E. P. Box and D. A. Pierce, "Distribution of residual autocorrelations in autoregressive-integrated moving average time series models," Journal of the American Statistical Association, vol. 65, no. 332, pp. 1509-1526, 1970.

[4] G. M. Ljung and G. E. P. Box, "On a measure of lack of fit in time series models," Biometrika, vol. 65, no. 2, pp. 297-303, 1978.

[5] A. I. McLeod and W. K. Li, "Diagnostic checking ARMA time series models using squared-residual autocorrelations," Journal of Time Series Analysis, vol. 4, no. 4, pp. 269-273, 1983.

[6] A. C. Monti, "A proposal for a residual autocorrelation test in linear models," Biometrika, vol. 81, no. 4, pp. 776-780, 1994.

[7] N. Katayama, "An improvement of the portmanteau statistic," Journal of Time Series Analysis, vol. 29, no. 2, pp. 359-370, 2008.

[8] N. Katayama, "On multiple portmanteau tests," Journal of Time Series Analysis, vol. 30, no. 5, pp. 487-504, 2009.

[9] D. Pierce, "Residual correlations and diagnostic checking in dynamic- disturbance time series models," Journal of the American Statistical Association, vol. 67, no. 339, pp. 636-640, 1972.

[10] A. I. McLeod, "On the distribution of residual autocorrelations in Box-Jenkins models," Journal of the Royal Statistical Society B: Methodological, vol. 40, no. 3, pp. 296-302, 1978.

[11] N. Davies, C. M. Triggs, and P. Newbold, "Significance levels of the Box-Pierce portmanteau statistic in finite samples," Biometrika, vol. 64, no. 3, pp. 517-522, 1977.

[12] C. Ansley and P. Newbold, "On the finite sample distribution of residual autocorrelations in autoregressive-moving average models," Biometrika, vol. 66, no. 3, pp. 547-553, 1979.

[13] G. M. Ljung, "Diagnostic testing of univariate time series models," Biometrika, vol. 73, no. 3, pp. 725-730, 1986.

[14] D. Peña and J. Rodríguez, "A powerful portmanteau test of lack of fit for time series," Journal of the American Statistical Association, vol. 97, no. 458, pp. 601-611, 2002.

[15] S. Chand and S. Kamal, "A comparative study of portmanteau tests for univariate time series models," Pakistan Journal of Statistics and Operations Research, vol. 2, no. 2, pp. 111-114, 2006.

[16] A. Joarder and M. Omar, "Exact distribution of the sum of two correlated chi-square variables and its application," Kuwait Journal of Science, vol. 40, no. 2, pp. 61-81, 2013. 


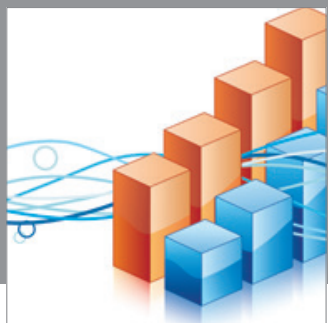

Advances in

Operations Research

mansans

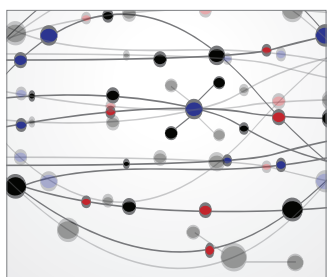

The Scientific World Journal
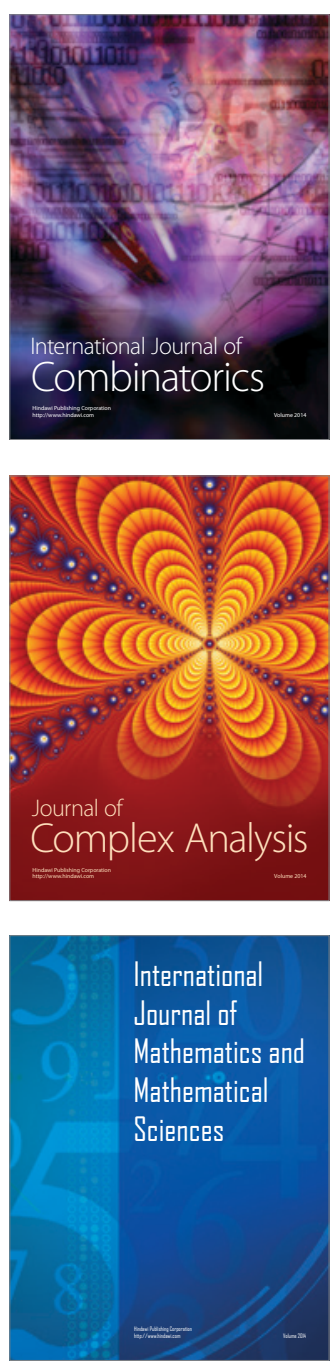
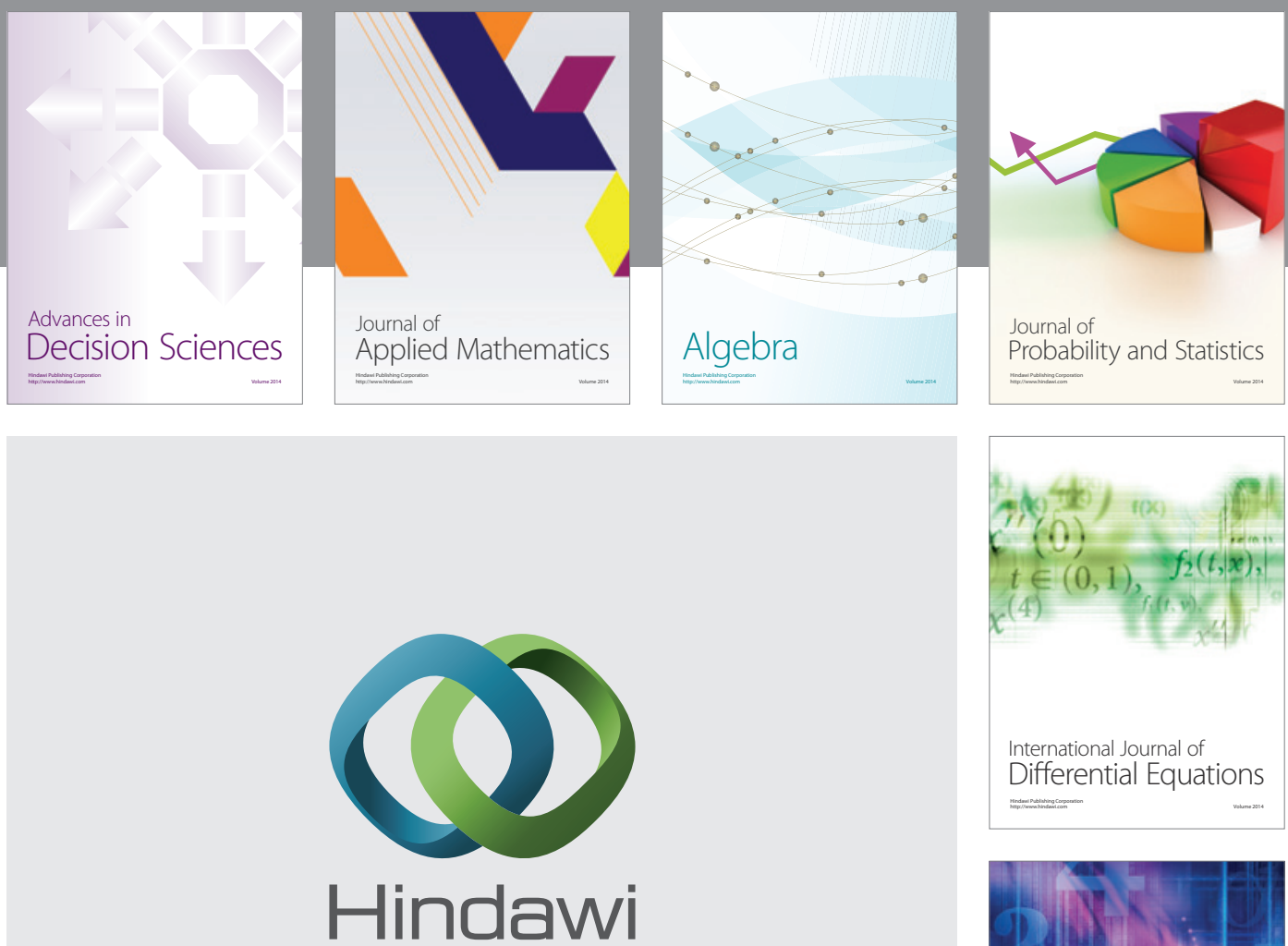

Submit your manuscripts at http://www.hindawi.com
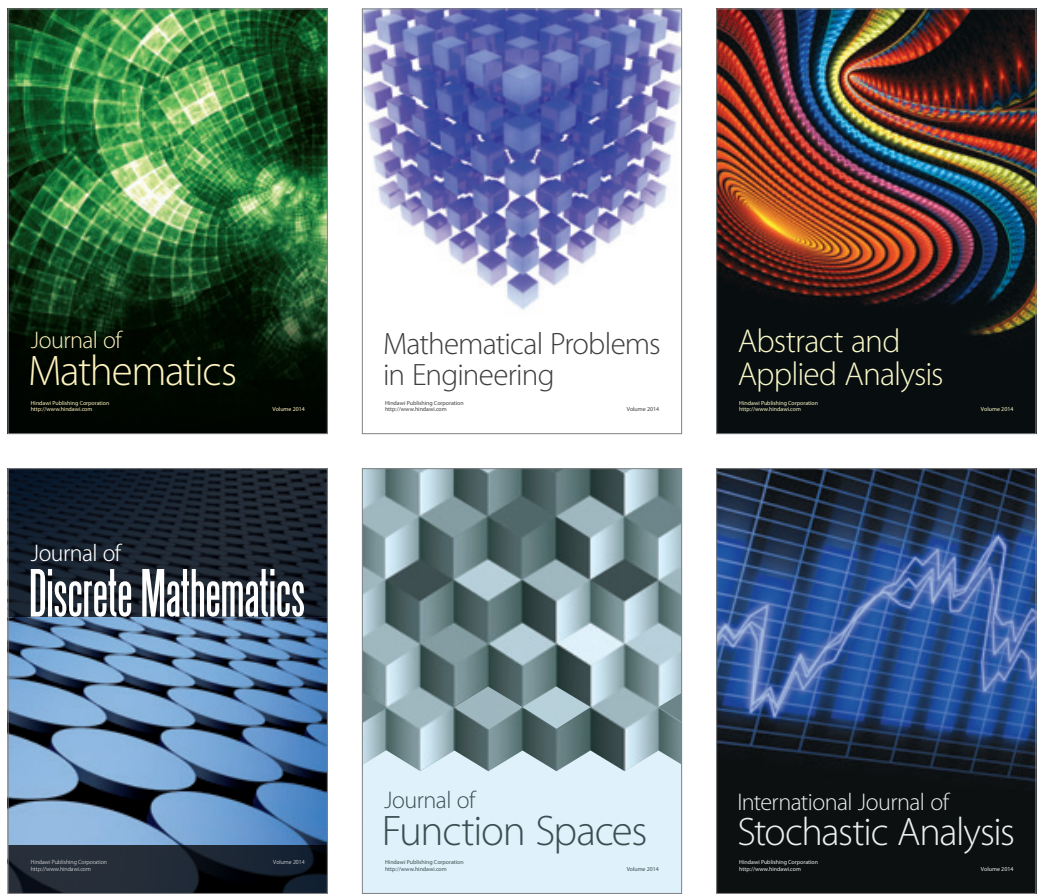

Journal of

Function Spaces

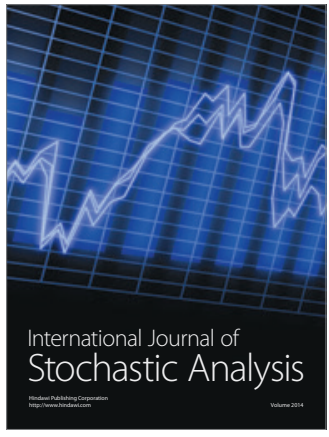

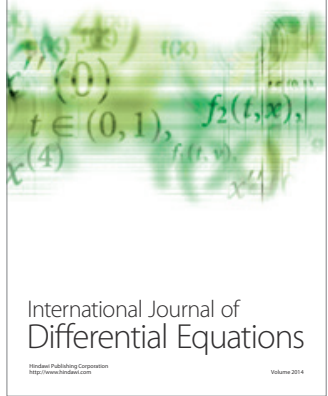
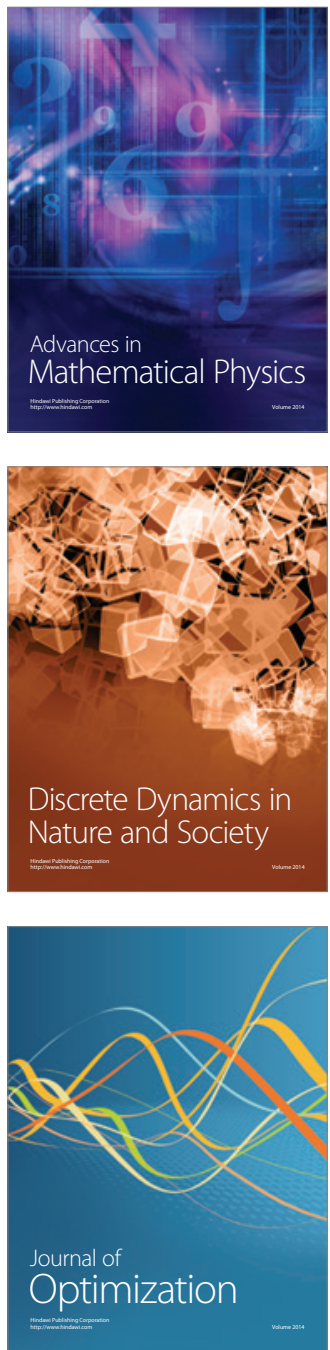